\title{
Applying the cost of generating force hypothesis to uphill running
}

Historically, several different approaches have been applied to explain the metabolic cost of uphill human running. Most of these approaches result in unrealistically high values for the efficiency of performing vertical work during running uphill, or are only valid for running up steep inclines. The purpose of this study was to reexamine the metabolic cost of uphill running, based upon our understanding of level running energetics and ground reaction forces during uphill running. In contrast to the vertical efficiency approach, we propose that during incline running at a certain velocity, the forces (and hence metabolic energy) required for braking and propelling the body mass parallel to the running surface are less than during level running. Based on this idea, we propose that the metabolic rate during uphill running can be predicted by a model, which posits that 1) the metabolic cost of perpendicular bouncing remains the same as during level running, 2) the metabolic cost of running parallel to the running surface decreases with incline, 3) the delta efficiency of producing mechanical power to lift the COM vertically is constant, independent of incline and running velocity, and 4) the costs of leg and arm swing do not change with incline. To test this approach, we collected ground reaction force (GRF) data for eight runners who ran thirty 30-second trials (velocity: 2.0-3.0 m/s; incline: 0-9). We also measured the metabolic rates of eight different runners for 17, 7-minute trials (velocity: 2.0-3.0 m/s; incline: 0-8ㅇ). During uphill running, parallel braking GRF approached zero for the 9 incline trials. Thus, we modeled the metabolic cost of parallel running as exponentially decreasing with incline. With that assumption, best-fit parameters for the metabolic rate data indicate that the efficiency of producing mechanical power to lift the center of mass vertically was independent of incline and running velocity, with a value of $\sim 29 \%$. The metabolic cost of uphill running is not simply equal to the sum of the cost of level running and the cost of performing work to lift the body mass against gravity. Rather, it reflects a constant cost of perpendicular bouncing, decreased costs of parallel braking and propulsion and of course the cost of lifting body mass against PeerJ reviewing PDF | (v2013:05:478:2:0:NEW 19 Jun 2014) 
gravity.

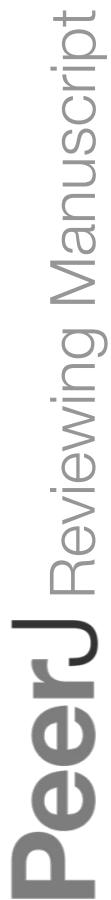

PeerJ reviewing PDF | (v2013:05:478:2:0:NEW 19 Jun 2014) 
1 Wouter Hoogkamer ${ }^{1}$, Paolo Taboga ${ }^{2}$ and Rodger Kram ${ }^{2}$

$2{ }^{1}$ Faculty of Human Movement Sciences, VU University, Amsterdam, the Netherlands ${ }^{2}$ Department

3 of Integrative Physiology, University of Colorado Boulder, CO. USA

4 Corresponding author:

5 Wouter Hoogkamer

6 Department of Kinesiology, KU Leuven

7 Tervuursevest 101 - bus 1501

83001 Heverlee, Belgium

9 Phone: 003216329065

10 Email: wouter.hoogkamer@faber.kuleuven.be 


\section{Introduction}

The energetic cost of running affects the behavior/performance of animals in nature, humans seeking fitness and athletes in competition. We believe that reasonable biomechanical explanations for the energetic cost of level running have been developed (Alexander \& Ker, 1990; Arellano \& Kram, 2014; Kram \& Taylor, 1990; Minetti \& Alexander, 1997; Roberts et al., 1998), but the world is not flat. We all know intuitively that running up even a slight incline is dramatically more exhausting, yet we lack a coherent biomechanical model for the energetic cost of uphill running.

In this paper, we develop and test a new model for the metabolic cost of uphill human running. Historically, several different approaches have been applied to this topic. Most of these approaches result in unrealistically high values for the efficiency of performing vertical work (Asmussen \& Bonde-Petersen, 1974; Lloyd \& Zacks, 1972; Pugh, 1971), or are only valid for running up steep inclines (Margaria et al., 1963; Margaria, 1968; Minetti et al., 2002) and not for running up inclines more typical of recreational/fitness running. The purpose of the current study was to reexamine the cost of uphill running, based upon our understanding of level running energetics (Kram \& Taylor, 1990; Roberts et al., 1998) and ground reaction forces during uphill running (Gottschall \& Kram, 2005).

First, we give an overview of how the energetics of uphill running have been approached in the past. Margaria and co-workers (Margaria et al., 1963; Margaria, 1968) calculated net mechanical efficiency of uphill running as:

Net mechanical efficiency $=$ vertical mechanical power $/$ net metabolic rate Here, the vertical mechanical power is the rate of performing work to raise the body mass $(m)$ against gravity $(g)$ : 
vertical mechanical power $=m \cdot g \cdot \sin (\theta) \cdot v$

Where $\theta$ is the incline in degrees and $v$ is the running velocity parallel to the incline. Margaria obtained the net metabolic rate by subtracting the basal metabolic rate from the metabolic rate during running. In level running, at a constant velocity, upon landing the body absorbs mechanical power (performs negative work) and then generates positive power (performs positive work) but no net external mechanical power is required because the negative and positive work quantities are opposite in sign but equal in magnitude (Cavagna, Saibene \& Margaria, 1964). Margaria (1968) proposed that the equal and opposite positive and negative external work can be considered to be wasted, since performing this work has a metabolic cost but does not propel the runner forward.

However, in uphill running, net positive external work and power are produced since the center of mass (COM) is raised against gravity. Margaria et al. (1963) hypothesized and demonstrated that on steeper inclines, the wasted external work decreases and the observed net mechanical efficiency approaches the same value as the efficiency of predominantly concentric exercise, such as cycle ergometry $(\sim 25 \%)$. It is important to note that this approach only results in such physiologically realistic efficiency values when the energetic cost of running is dominated by the work needed to raise the COM (i.e. at steep inclines) (Minetti et al., 2002). For running up inclines more typical of recreational/fitness running the net mechanical efficiencies calculated are much lower than the values for concentric muscle contractions (Smith, Barclay \& Loiselle, 2005). 
Published values for vertical efficiency range from 30\% for red kangaroos (Kram \& Dawson, 1998 ) to $\sim 46 \%$ for humans (Asmussen \& Bonde-Petersen, 1974; Lloyd \& Zacks, 1972; Pugh, 1971), to values near 50\% (walking turtles (Zani \& Kram 2008)) or even higher ( $60 \%$ for mice and $66 \%$ for chimpanzees (Taylor, Caldwell \& Rowntree, 1972)). In running, these efficiency values, which are much higher than isolated muscle contraction efficiency, have been attributed to elastic energy storage and reutilization in muscle-tendon complexes (Asmussen \& BondePetersen, 1974; Lloyd \& Zacks, 1972; Cooke et al., 1991). But, as emphasized by Roberts et al. (1997), the increase in potential energy of the body in uphill locomotion can only be done by active concentric muscle work, since passive elastic mechanisms simply return energy stored previously in a step. Thus, these high efficiency values remain enigmatic.

Alternatively, Minetti, Ardigò \& Saibene (1994) developed a model which assumed that the metabolic cost can be predicted completely based on measures of mechanical work. In their model, internal work (due to the kinetic energy changes of body segments relative to the body $\mathrm{COM}$ ), positive external work and negative external work were each assumed to be performed with a separate efficiency value. Minetti, Ardigo \& Saibene's model (1994) also estimates the amount elastic energy storage and release, however the costs of muscle force production to generate tension to allow this energy storage and release is not taken into account.

Although we believe that the cost of generating force to support body weight is the major determinant of the metabolic cost of level running (for review, see Arellano \& Kram, 2014; Kram, 2000), none of the models for uphill running explicitly include this cost. Briefly, the cost of generating force hypothesis posits that in running the muscles primarily act to generate tension that allows the tendons to store and return elastic energy. Muscles consume energy whenever they generate tension, regardless of whether they perform work. The cost of generating force to 
support body weight has been found to be inversely proportional to the foot-ground contact time, presumably because generating force more rapidly requires faster and less economical muscle fibers (Roberts et al., 1998).

In this study, we introduce a model for the metabolic cost of uphill running which combines the cost of generating force and the cost of performing mechanical work approaches. Our overall view is that the net metabolic cost of running is comprised of the costs of generating force to support body weight, braking and propelling body mass in the forward (parallel) direction, swinging the legs and arms and maintaining balance (Arellano \& Kram, 2011; Arellano \& Kram, 2014; Chang \& Kram, 1999; Farley \& McMahon, 1992; Kram \& Taylor, 1990; Modica \& Kram, 2005; Moed \& Kram, 2005; Teunissen, Grabowski \& Kram, 2007). For level running, obviously body weight must be dynamically supported in the vertical direction, but for uphill running, we prefer to call this term the cost of "perpendicular bouncing" to emphasize that the metabolic power required to lift the COM vertically is not included in that term (Figure 1). This approximation introduces only a small error because the perpendicular component is only slightly less than the vertical component, for example, the cosine of 9 degrees equals 0.988 . Furthermore, Gottschall \& Kram (2005) observed that both the perpendicular active force peaks and the contact times during uphill running (up 3, 6 and $9^{\circ}$ ) were not significantly different from those during level running. Thus, based on the cost of generating force hypothesis, the cost of perpendicular bouncing should not change with incline. So, in uphill running, the net metabolic rate should be equal to the sum of the rates of metabolic energy consumption for perpendicular bouncing, braking and propelling body mass parallel to the surface, swinging the legs and arms and, of course, raising of the COM vertically. In Figure 1 parallel running refers to the task of running parallel to the surface whether that surface is level or inclined. The task of parallel 
running intrinsically requires bouncing perpendicular to the surface and that bouncing incurs a metabolic cost.

\section{<Figure 1 approximately here $>$}

In contrast to the vertical efficiency approach, we propose that at a certain velocity the metabolic rate required for braking and propelling the body mass parallel to the running surface is less during inclined running (compared to level running), because there is less braking (negative external work) and thus less wasted work (Margaria, 1968; Minetti, Ardigò \& Saibene, 1994). Gottschall \& Kram (2005) quantified how in uphill running the braking Ground Reaction Forces (GRFs) parallel to the running surface decrease with steeper inclines. The propulsive GRFs parallel to the running surface are greater during uphill running, but the majority of the propulsive GRF impulse parallel to the running surface compensates for the gravitational braking impulse parallel to the surface $m \cdot g \cdot \sin (\theta) \cdot t_{\text {step }}$, where $t_{\text {step }}$ is the time between two consecutive foot strikes. During steeper incline running, most of the propulsive parallel GRF impulse is required to overcome the component of the gravitational braking impulse parallel to the surface. Thus, only a small part of the parallel propulsive GRF impulse is compensating for the braking GRF impulse (Figure 2). Although initially counterintuitive, the metabolic costs of both braking and propelling forces, parallel to the running surface, should decrease during uphill running. By taking that into account, the efficiency of producing mechanical power to lift the COM vertically should be closer to the efficiency of concentric muscle contractions.

\section{$<$ Figure 2 approximately here $>$}

123 Based on these ideas, we propose that the metabolic rate during uphill running can be predicted 124 by a model, which posits that 1) the metabolic cost of perpendicular bouncing remains the same 125 as during level running, 2) the metabolic cost of running parallel to the running surface decreases 
with incline, 3) the delta efficiency (Gaesser \& Brooks, 1975) of producing mechanical power to lift the COM vertically $\left(E f f_{v C O M}\right)$ is constant, independent of incline and running velocity, and 4) the costs of leg and arm swing do not change with incline. We expect Eff $_{v C O M}$ to be similar to the delta efficiency of cycling ( 25-30\%) (Gaesser \& Brooks, 1975; Bijker, De Groot \& Hollander, 2001). To test these ideas, we measured GRFs for level and a range of uphill running inclines (1$\left.9^{\circ}\right)$ for a range of velocities $(2.0-3.0 \mathrm{~m} / \mathrm{s})$. Additionally, we measured the metabolic rate during uphill running for a feasible range of grades at the same velocities $\left(0-8^{\circ}\right.$ at $2.0 \mathrm{~m} / \mathrm{s} ; 0-4^{\circ}$ at 2.5 and $3.0 \mathrm{~m} / \mathrm{s}$ ).

\section{Materials \& Methods}

\section{Ground Reaction Forces}

For this part of the study, eight participants ran on a force treadmill (Treadmetrix, Park City, UT, USA). Six males and two females participated $(31.2 \pm 11.0 \mathrm{yr}, 177.6 \pm 7.1 \mathrm{~cm}, 69.5 \pm 7.9 \mathrm{~kg}$; all mean \pm SD). The participants gave written informed consent and the testing protocol was approved by the University of Colorado Institutional Review Board (13-0710).

Each trial lasted 30 seconds and the first 20 steps after the first 10 seconds were analyzed. Forces were collected at $1000 \mathrm{~Hz}$. Before each trial forces were zeroed by regulating the amplifiers (MSA-6 MiniAmp, AMTI Watertown, MA, USA) and the acquisition software (Vicon Nexus, Vicon Motion Systems Ltd., Oxford, UK ). Signals were digitally filtered using a first-order Butterworth filter (pass band frequency of $35 \mathrm{~Hz}$ and stop band frequency of $50 \mathrm{~Hz}$ ) implemented in a Matlab script (Mathworks Inc., USA). A $10 \mathrm{~N}$ threshold was used to determine the instants of 
foot strike and toe-off. Per step, we calculated the average braking and propelling GRF impulses parallel to the running surface by integrating all negative (braking) or positive (propelling) values during each ground contact. We used the time between two consecutive foot strikes $t_{\text {step }}$ (for example from left foot strike to right foot strike) to calculate the average gravitational impulses parallel to the surface:

$I_{-}$Gravity $_{\text {parallel }}=m \cdot g \cdot \sin (\theta) \cdot t_{\text {step }}$

We defined the total propelling impulse per step as the propelling GRF impulse minus the component of the gravitational impulse, all parallel to the running surface:

$I_{-} G R F_{\text {propelling_total }}=I_{-} G R F_{\text {propelling }}-I_{-}$Gravity parallel

Summation of the absolute values of the braking impulse and of the total propulsive impulse per step gave the value of the wasted GRF impulse per step:

$I_{-} G R F_{\text {wasted }}=I_{-} G R F_{\text {braking }}+I_{-} G R F_{\text {propelling_total }}$

For level running, the wasted GRF impulse per step equals the summation of the absolute values of the braking impulse and the propulsive impulse per step, similar to the concept of wasted work per step as introduced by Margaria (1968). We note that not all of the wasted impulse is actively done by muscle length changes; a substantial part is likely provided through passive elastic storage and return. Based on earlier studies with kinetic (Gottschall \& Kram, 2005) and kinematic (Minetti, Ardigò \& Saibene, 1994) measurements of uphill running and the fact that $I_{-} G R F_{\text {wasted }}$ cannot be negative, we hypothesized that $I_{-} G R F_{\text {wasted }}$ decreases exponentially to zero for steeper inclines:

$I_{-} G R F_{\text {wasted }}=I \_G R F_{\text {wasted_level }} e^{-\gamma \cdot \sin (\theta)}$

Parameter $I_{-} G R F_{\text {wasted_evel }}$ represents the value of the wasted GRF impulse per step during level running. The decay constant $\gamma$ determines how steeply $I \_G R F_{\text {wasted }}$ decreases with incline (expressed as $\sin (\theta))$. 
Metabolic measurements

171 We recruited a different eight participants for this part of the study (4 males and 4 females,

$17226.2 \pm 4.0 \mathrm{yr}, 174.3 \pm 12.4 \mathrm{~cm}, 67.3 \pm 11.8 \mathrm{~kg}$; all mean $\pm \mathrm{SD})$. All participants had extensive

173 treadmill running experience and had recently run a $5 \mathrm{~km}$ race in less than 20 minutes $(18: 28 \pm 52$

$174 \mathrm{sec}$; mean $\pm \mathrm{SD}$ ). Based on pilot testing, we were confident that for this caliber of runner, the most

175 demanding test condition would be submaximal. We applied this 20 -minute $5 \mathrm{~km}$ criteria to 176 ensure that the energy supply during our experimental trials was predominately oxidative and to 177 avoid fatigue effects. The participants gave written informed consent that followed the guidelines 178 of the University of Colorado Institutional Review Board (0606.29).

179 Participants completed different sessions on two separate days. They ran a total of 17 different conditions on a classic Quinton 18-60 treadmill with adjustable velocity and incline. Note that we modified this treadmill so that we had calibrated, digital electronic readouts for velocity and incline. On the first day, participants ran at a velocity of $2.0 \mathrm{~m} / \mathrm{s}$ at seven different inclines ranging from 0 to $8^{\circ}$. The second day consisted of five trials at both $2.5 \mathrm{~m} / \mathrm{s}$ and $3.0 \mathrm{~m} / \mathrm{s}$ at inclines ranging from 0 to $4^{\circ}$ (for a complete list of the trials, refer to Table 2 in the Results section). We measured the rates of oxygen consumption and carbon dioxide production during these 7-minute trials. Each experimental day started with determining the body mass of the participant. We then determined metabolic rate during a 7-minute standing trial. This was followed by a 10-minute warm-up of level running at $2.0 \mathrm{~m} / \mathrm{s}$ or $2.5 \mathrm{~m} / \mathrm{s}$, for the first and second day, respectively. During warm-up, participants breathed through the expired-gas analysis system to allow acclimatization. For each running velocity, the different incline conditions were randomized to prevent order effects. 
192 We measured the rates of oxygen consumption $\left(\mathrm{VO}_{2}\right)$ and carbon dioxide production $\left(\mathrm{VCO}_{2}\right)$

193 using an open-circuit expired-gas analysis system (True One 2400, Parvo Medics, Salt Lake City,

194 UT, USA). We calibrated the gas analyzers before each test using reference gases. The flow-rate

195 transducer was calibrated using a 3 liter syringe (Rudolph Inc., Kansas City, MO, USA). Each

196 trial lasted 7 minutes based on pilot data showing that steady state was reached in less than 5

197 minutes during the different trials. We averaged $\mathrm{VO}_{2}, \mathrm{VCO}_{2}$ and respiratory exchange ratios

198 (RER) for the last 2 minutes of each trial. Rest periods of at least 4 minutes occurred between the

199 trials. During the rest periods, the treadmill was adjusted to the incline and velocity of the

200 following trial.

201 Calculations

202 To fit a generic curve to the wasted impulse data (eq. 6), we first normalized the impulse data to

203 body mass and divided the values by running velocity, similar to the cost of transport concept

204 (see below). Mechanical vertical COM power (in Watts) was calculated using belt velocity and

205 incline (similar to equation 2):

206 Mechanical vertical COM power $=m \cdot g \cdot \sin (\theta) \cdot v$

207 Where $\theta$ is the incline in degrees and $v$ is velocity in $\mathrm{m} / \mathrm{s}$. Metabolic rates (in $\mathrm{W} / \mathrm{kg}$ ) were

208 calculated from respiratory measurements using the Brockway equation (Brockway, 1987). Net

209 metabolic power was calculated as running metabolic rate minus the standing metabolic rate. We

210 calculated the traditional values of delta efficiency of producing mechanical power to lift the

211 COM vertically as the ratio of mechanical vertical COM power to the difference in metabolic rate

212 between level running and running on incline at the same velocity (Gaesser \& Brooks, 1975).

213 Net metabolic Cost of Transport (CoT) is the net metabolic cost per unit distance traveled parallel

214 to the running surface. It is calculated by dividing the net metabolic rate by the running velocity 
215 and is expressed in $\mathrm{J} /(\mathrm{kg} \cdot \mathrm{m})$. Cost of Transport values allowed us to develop a generalized

216 equation, independent of running velocity. Based on the general concepts underlying our uphill

217 running model, we generated a custom equation and fitted this to the data to calculate the

218 parameters resulting in the best fit (see below). The format of the equation is:

$219 \operatorname{Net} \operatorname{CoT}(\mathrm{J} /(\mathrm{kg} \cdot \mathrm{m}))=A+B \cdot e^{-\lambda \cdot \sin (\theta)}+\frac{g}{E f f_{v \operatorname{COM}}} \cdot \sin (\theta)$

220 In this equation, the CoT of parallel running is represented by $A+B \cdot e^{-\lambda \cdot \sin (\theta)}$. We postulated that

221 the cost of parallel running decreases exponentially with incline. We expected that at steep

222 inclines, where $I_{-} G R F_{\text {wasted }}$ equals zero, the cost of braking and propelling would be reduced to

223 zero and that the cost of parallel running would consist of only the costs of perpendicular

224 bouncing, leg swing and arm swing. In terms of our model, the first term $A$ represents the CoT

225 related to perpendicular bouncing, leg swing and arm swing. Parameter $B$ represents the CoT for

226 braking and propelling during level running. For inclined running, the CoT for braking and

227 propelling parallel to the running surface decreases exponentially with the sine of the incline

228 angle $\theta: \mathrm{CoT}_{\text {braking } / \text { ropelling }}=B \cdot e^{-\lambda \cdot \sin (\theta)}$. The decay constant $\lambda$ determines how steeply the

$229 \mathrm{CoT}_{\text {braking/propelling }}$ decreases with $\sin (\theta)$. Logically, the $\mathrm{Co}_{\text {braking/propelling }}$ decreases proportionally to

230 the wasted GRF impulse per step $I_{-} G R F_{\text {wasted }}$, i.e. that $\lambda$ in equation 8 is equal to $\gamma$ in equation 6.

231 The CoT of producing mechanical power to lift the COM vertically is represented by the third

232 term in equation 8 . To relate the mechanical vertical COM power (equation 7) to the metabolic

233 CoT, it should be divided by body mass, velocity and the efficiency of producing mechanical

234 power to lift the COM vertically, resulting in $\frac{g}{E f f_{v C O M}} \cdot \sin (\theta)$. 
236 We present all results in the text as mean values \pm SD. We used a traditional level of significance

$237(\alpha=0.05)$ for all statistical tests. To test for significant differences between the three tested 238 running velocities and between different angles, we applied two-way analyses of variance

239 (ANOVAs) on the impulse, step frequency and contact time data. We applied the non-linear least 240 squares method to fit non-linear curves on the data and the linear least squares method to fit lines. 241 We utilized $r^{2}$ to evaluate goodness of fit.

\section{Results}

243 Ground Reaction Forces

244 For running at a velocity of $2.0 \mathrm{~m} / \mathrm{s}$, the braking GRF impulse per step, parallel to the running 245 surface, normalized to body mass and divided by the running velocity, decreased significantly 246 from -0.128 for level running to -0.003 for running up a $9^{\circ}$ incline. For $2.5 \mathrm{~m} / \mathrm{s}$ and $3.0 \mathrm{~m} / \mathrm{s}$

247 similar decreases were observed (Table 1). For two participants, we could not analyze the kinetic 248 data at $2 \mathrm{~m} / \mathrm{s}$ since they "ran" without a clear aerial phase at this velocity. As such, their stance 249 phases partially overlapped, invalidating the assessment of the braking and propelling impulses 250 during each stance phase. In addition, for the same reason, we excluded 4 separate trials for other 251 participants. Similar to the braking impulse, the total propelling impulse per step, parallel to the 252 running surface, and the wasted GRF impulse per step $\left(I_{-} G R F_{\text {wasted }}\right)$, also decreased with incline 253 (Table 1; Figure 3; individual trial data is contained in the supplemental material). Recall that 254 total propelling impulses were calculated as the propelling GRF impulse parallel to the surface 
255 minus the component of the gravitational impulse parallel to the running surface. Summation of

256 the absolute values of the braking impulse and of the total propelling impulse per step gave

257 I_GRF $F_{\text {wasted. }}$ In line with our hypothesis, $I$ GRF $F_{\text {wasted }}$ values decreased exponentially with incline.

258 Curve fitting of equation 6 to the GRF data resulted in best-fit parameter values of $I_{-} G R F_{\text {wasted_level }}$

$259=0.1208$ and $\gamma=18.24$, with $r^{2}=0.89$ (Figure 3 ). Fitting a linear equation to the data resulted in a

260 lower correlation between the data and the fit $\left(\mathrm{r}^{2}=0.79\right)$ and implied negative $I$ GRF $F_{\text {wasted }}$ values

261 for inclines steeper than about 8 degrees.

$262<$ Figure 3 approximately here $>$

263 The step frequency increased significantly with incline and with running velocities (Table 1). In 264 contrast, contact times were similar between inclines $(\mathrm{p}=0.7)$ and decreased significantly with 265 velocity (Table 1).

266 <Table 1 approximately here>

267 Metabolic measurements

268 In Table 2 we present the mean rates of oxygen consumption $\left(\mathrm{VO}_{2}\right)$ and metabolic energy

269 consumption (individual trial data is contained in the supplemental material). For all participants,

270 RER $(0.86 \pm 0.05$; range $0.74-0.96)$ was less than 1.0 for all trials, indicating that the metabolic

271 energy was derived primarily from oxidative sources. The mean metabolic rate for standing was

$272 \quad 1.53 \pm 0.08 \mathrm{~W} / \mathrm{kg}$.

$273<$ Table 2 approximately here>

274 Decreasing cost of parallel running 
275 Net metabolic Cost of Transport (CoT) data for all participants are shown in Figure 4A for 276 different inclines and velocities. The net CoT data are plotted versus the sine of the incline angle $277 \theta$ on the primary horizontal axis (at the bottom) because the vertical power is proportional to the 278 sine of the incline angle. The net CoT is the net metabolic cost expressed per unit distance 279 traveled parallel to the running surface.

280 We set $\lambda$ in our model (eq. 8) to be equal to $\gamma$ (from eq. 6) and calculated the best fit to the 281 metabolic data. The parameter of the best fit with $\lambda=\gamma=18.24$ were $A=2.70, B=0.674$ and 282 $E f f_{v C O M}=29.4 \%$ with $\mathrm{r}^{2}=0.97$. The best-fit curve is shown in Figure 4A. In Figure 4B this bestfit curve is labeled Net $\mathrm{CoT}_{\text {total }}$ as it includes all terms of equation 8, i.e. the CoT of parallel running and the CoT of producing mechanical power to lift the COM vertically. The net CoT data are shown as mean values for each running velocity in this figure (Figure 4B). The metabolic CoT of producing mechanical power to lift the COM vertically is also shown (labeled $\mathrm{CoT}_{\text {vertical }}$ ). This CoT was calculated based on $E f f_{v C O M}=29.4 \%$. In our model, the CoT of parallel running is represented by $A+B \cdot e^{-\lambda \cdot \sin (\theta)}$, and this cost is shown in Figure $4 \mathrm{C}$ for $A=2.70, B=0.674$ and $\lambda=$ 18.24. Metabolic data points were calculated by subtracting the calculated metabolic CoT of producing mechanical power to lift the COM vertically from the net CoT. This resulted in the following equation:

$292 \operatorname{Net} \operatorname{CoT}(\mathrm{J} /(\mathrm{kg} \cdot \mathrm{m}))=2.70+0.674 \cdot e^{-18.24 \cdot \sin (\theta)}+\frac{g}{0.294} \cdot \sin (\theta)$ (eq. 9)

$293<$ Figure 4 approximately here $>$

294 Note that the best-fit regression for the net CoT versus the sine of the incline angle $\theta$, using 295 equation 8, is fairly insensitive to changes in the parameters. For instance, curve fitting of 
equation 8 with $\lambda$ as a free parameter resulted in $A=1.16, B=2.20, \lambda=7.60$ and $E f f_{v C O M}=24.9 \%$ and produced a similar goodness of fit: $\mathrm{r}^{2}=0.97$.

\section{Discussion}

In this study, we quantified the ground reaction forces and metabolic cost of uphill human running and introduced a new model to interpret our results. This is the first model for uphill running that incorporates the cost of generating force concept. We have found that the metabolic rate during uphill running can be predicted by a model which posits that 1 ) the metabolic cost of perpendicular bouncing remains the same as during level running, 2) the metabolic cost of running parallel to the running surface decreases with incline, 3) the delta efficiency of producing mechanical power to lift the COM vertically $\left(E f f_{v C O M}\right)$ is constant, independent of incline and running velocity, and 4) the costs of leg and arm swing do not change with incline.

\section{Ground Reaction Forces}

The GRF data confirmed that the wasted braking and propulsive impulses per step decrease exponentially with incline supporting our contention that the metabolic cost of parallel running decreases with incline. Based on this, we generated a general model for the metabolic cost of uphill running.

\section{Metabolic cost of uphill running}


313 In line with earlier observations of net mechanical efficiency values approaching the efficiency of

314 concentric contracting muscles on steeper inclines (Margaria, 1968; Margaria et al., 1963), our

315 model assumes that the efficiency of producing mechanical power to lift the COM vertically

$316\left(E f f_{v C O M}\right)$ is constant, independent of incline and running velocity, and physiologically realistic.

317 Our method offers an alternative to the model by Minetti and co-workers (Minetti, Ardigò \&

318 Saibene, 1994; Minetti et al., 2002) which assumed that the metabolic cost can be predicted

319 completely based on measures of mechanical work. In contrast, our model combines the cost of

320 generating force to support the runner's body weight and the cost of performing mechanical work

321 to lift the COM. In our approach, the different terms in the model each represent different

322 elements of the CoT of uphill running. Unfortunately, the CoT of each of these elements cannot

323 be measured independently. Therefore, we constructed a biomechanical realistic model and

324 applied a fitting procedure to calculate the parameters needed.

325 Metabolic cost of parallel running

326 According to equation 9 , for level running $(\theta=0 ; \sin (\theta)=0)$, about $80 \%$ of the net metabolic

327 CoT would be attributed to weight support (perpendicular bouncing), leg swing and arm swing,

328 while $20 \%$ would be attributed to braking and propelling the COM. These number relate well

329 with earlier studies on the cost of supporting body weight (at most $74 \%$ of the net cost of

330 running; Teunissen, Grabowski \& Kram, 2007) and of leg swing (only 10\% of the net cost of

331 running; Moed \& Kram, 2005), which sum up to $\sim 84 \%$ of the net metabolic cost attributable to

332 weight support and leg swing.

333 In our model, the CoT related to perpendicular bouncing, leg swing and arm swing is independent

334 of incline. However, step frequency increased slightly with incline, which could result in higher 
335 values for "internal work" (Minetti, 1998) or joint mechanical power (Swanson \& Caldwell,

336 2000). We estimated mechanical internal work values (in $\mathrm{J} /(\mathrm{kg} \cdot \mathrm{m})$ ) based on step frequency, duty

337 factor and velocity as per the Minetti equation (1998), using different values for factor $q$ for level

338 and uphill running (Nardello, Ardigò \& Minetti, 2011). These estimates of mechanical internal

339 work increased both with incline and running velocity. Although the Minetti equation (1998)

340 suggests that internal power would increase by $37 \%$ between 2.0 and $3.0 \mathrm{~m} / \mathrm{s}$, we did not observe

341 any change in the overall metabolic CoT. Similar increases in the internal mechanical power were

342 estimated between level and uphill running (32 - 33\%, for our range of velocities). It is unclear

343 how these mechanical internal work estimates relate to the metabolic CoT, because of

344 overestimations of internal work related to the ballistic pendulum-like part of the swing phase of

345 the limbs (Alexander, 1989). Furthermore, Nardello, Ardigò \& Minetti (2011) reevaluated the

3461998 Minetti equation for humans of both sexes, for different age groups, running at different

347 velocities and inclines and they observed no increase in measured internal work as function of

348 incline for velocities below $2.78 \mathrm{~m} / \mathrm{s}$. Additionally, evidence from our laboratory suggests that the

349 metabolic cost of leg swing in human running is relatively small, $\sim 10-20 \%$ of net metabolic cost

350 of running (Modica \& Kram, 2005; Moed \& Kram, 2005). Finally, guinea fowl blood flow data

351 suggest that the majority of the increased energy expenditure in uphill running is used by stance

352 phase muscles (Rubenson et al., 2006). Thus, for simplicity in our model, we assumed that the

353 cost of leg swing is independent of incline.

354 It is difficult to estimate the metabolic cost of arm swing. Experiments that restrict arm swing

355 increase the cost of running by at least 3\% (Arellano \& Kram, 2014) suggesting that arm swing

356 produces a net energy savings rather than a net cost. In any case, it seems unlikely that the

357 metabolic cost or savings due to arm swing at a certain running speed would change greatly 
during uphill running. Thus, we subsume the cost of arm swing into the cost of perpendicular bouncing and assume that it does not change.

Metabolic cost of producing mechanical power to lift the COM vertically

The CoT of producing mechanical power to lift the COM vertically increases linearly with $\sin (\theta)$, proportional to the mechanical vertical COM power. This is a direct consequence of our assumption that the efficiency of producing mechanical power to lift the COM vertically (Eff $\left.f_{v C O M}\right)$ is constant, independent of incline and running velocity. The efficiency of producing mechanical power to lift the COM vertically $\left(E f f_{v C O M}\right)$, according the best fit of our model was $29.4 \%$. This value is in the same range as earlier reported values of similar measures of whole body efficiency in cycling. Gaesser \& Brooks (1975) defined work efficiency as work accomplished divided by the energy expended above that in cycling without a load. They found values ranging from 25.4 to $30.3 \%$ for increasing cadence and power output. Bijker, De Groot \& Hollander (2001) reported a mean delta efficiency (delta work accomplished over delta energy expended) of $25.8 \%$ in ergometer cycling. In contrast, Margaria's net mechanical efficiency (vertical mechanical power / net metabolic rate) values were rather low ( $\sim 9-16 \%$; Minetti et al., 2002) for running up inclines typical of recreational running. Alternatively, the traditional vertical efficiency (vertical mechanical power / difference in metabolic rate between locomotion on an incline and level locomotion at the same velocity) and similarly calculated measures result in high values ( $\sim 36-$ 46\%; Asmussen \& Bonde-Petersen, 1974; Bijker, De Groot \& Hollander, 2001; Cooke et al., 1991; Lloyd \& Zacks, 1972; Pugh, 1971). 
Our study has several limitations worthy of mention. As discussed earlier, we performed the two parts of the study (GRF and metabolic data collection) with two different groups of participants. We acknowledge this as a limitation of the study, however, because our model parameters were calculated using regression equations for group data we consider this not to be a serious concern. Further, we are not attempting to make subject specific conclusions, rather we are seeking general principles. Overall, we were limited by the aerobic capacity of the participants. We tried to include a broad range of velocities and inclines, but we were restricted by our aim to consider only conditions that could be run at truly submaximal intensities by all our participants. Although, we did not quantify the elastic energy storage and reutilization, we accounted for this by introducing the cost of perpendicular bouncing, which we assumed to be independent of incline and proportional to velocity.

The, overall, promising agreement between the experimental data and the equations based on the assumptions underlying our approach, call for further validation of this approach in future studies. Addressing any effects on cost of potential changes in internal work (CoT of leg swing), mechanical joint work and joint posture could refine the accuracy of and increase the confidence in our approach. It would be interesting to study the energetics of uphill walking with the same approach as we have done here for running. More insights into the energetics of downhill running may be gained with our approach. Of course, our concept of decreased parallel braking impulses would need to be reversed.

\section{Conclusions}


Overall, we postulate that the metabolic rate during uphill running is not simply equal to the sum of the cost of level running and the cost of performing work to lift the body mass against gravity. Rather, our new approach suggests that the metabolic cost of running at a certain velocity, parallel to the running surface, decreases with incline, and that the efficiency of producing mechanical power to lift the COM vertically is constant, independent of incline and running velocity. With this approach, we have been able to model the observed metabolic rates during uphill running at different velocities and inclines.

\section{Acknowledgements}

We thank Dr. Maarten Bobbert and members of the Locomotion Lab of the University of Colorado Boulder for insightful comments and suggestions.

\section{References}

Abbott BC, Bigland B, Richie JM. 1952. The physiological cost of negative work. Journal of Physiology 117:380-390

Alexander RM. 1989. Optimization and gaits in the locomotion of vertebrates. Physiological Reviews 69:1199-1227

Alexander RM. 1992. A model of bipedal locomotion on compliant legs. Philosophical Transactions of the Royal Society B: Biological Sciences 338:189-198

Alexander RM, Ker RF. 1990. Locomotion. Running is priced by the step. Nature 346:220-221 American College of Sports Medicine. 2009. ACSM's Guidelines for Exercise Testing and Prescription, 8th ed. Philadelphia: Lippincott Williams \& Wilkins. 158

Arellano CJ, Kram R. 2014. On the metabolic cost of human running: is swinging the arms worth it? Journal of Experimental Biology In press 
Arellano CJ, Kram R. 2014. Partitioning the Metabolic Cost of Human Running: A Task-by-Task

Approach. Integrative and Comparative Biology [Epub ahead of print] doi:10.1093/icb/icu033

Asmussen E, Bonde-Petersen F. 1974. Apparent efficiency and storage of elastic energy in human muscles during exercise. Acta Physiologica Scandinavica 92:537-545

Bijker KE. 2003. Metabolic cost of running and walking. Dissertation, VU University of Amsterdam

Bijker KE, De Groot G, Hollander AP. 2001. Delta efficiencies of running and cycling. Medicine and Science in Sports and Exercise 33:1546-1551

Brockway JM. 1987. Derivation of formulae used to calculate energy expenditure in man.

Human Nutrition - Clinical Nutrition 41:463-471

Brooks GA, Fahey TD, Baldwin KM. 2005. Exercise Physiology: Human Bioenergetics and its Applications, $4^{\text {th }}$ ed. New York: McGraw-Hill. 698-700

Cavagna GA, Saibene FP, Margaria R. 1964. Mechanical work in running. Journal of Applied Physiology 19:249-256

Chang Y-H, Kram R. 1999. Metabolic cost of generating horizontal forces in running. Journal of Applied Physiology 86:1657-1662

Cooke CB, McDonagh MJN, Nevill AM, Davies CTM. 1991. Effects on load on oxygen intake in trained boys and men during treadmill running. Journal of Applied Physiology 71:1237-1244

Farley CT, McMahon TA. 1992. Energetics of walking and running - insights from simulated reduced-gravity experiments. Journal of Applied Physiology 73:2709-2712

Full RJ, Tullis A. 1990. Energetics of ascent: insects on inclines. Journal of Experimental Biology 149:307-317

Gaesser GA, Brooks GA. 1975. Muscular efficiency during steady-rate exercise: effects of speed and work rate. Journal of Applied Physiology 38:1132-1139

Gottschall JS, Kram R. 2005. Ground reaction forces during downhill and uphill running. 
Journal of Biomechanics 38:445-452

Kram R. 2000. Muscular force or work: what determines the metabolic energy cost of running?

Exercise and Sport Sciences Reviews 28:138-143

Kram R, Dawson TJ. 1998. Energetics and biomechanics of locomotion by red kangaroos

(Macropus rufus). Comparative Biochemistry and Physiology 120B:41-49

Kram R, Taylor CR. 1990. Energetics of running: a new perspective. Nature 346:265-267

Lloyd BB, Zacks RM. 1972. The mechanical efficiency of treadmill running against a horizontal impeding force. Journal of Physiology 223:355-363

Margaria R. 1968. Positive and negative work performances and their efficiencies in human locomotion. Internationale Zeitschrift für Angewandte Physiologie 25:339-351

Margaria R, Ceretelli P, Aghemo P, Sassi G. 1963. Energy cost of running. Journal of Applied Physiology 18:367-370

Minetti AE. 1998. A model equation for the prediction of mechanical internal work of terrestrial locomotion. Journal of Biomechanics 31:463-468

Minetti AE, Alexander RM. 1997. A theory of metabolic costs for bipedal gaits. Journal of Theoretical Biology 186:467-476

Minetti AE, Ardigò LP, Saibene F. 1994. Mechanical determinants of the minimum energy cost of gradient running in humans. Journal of Experimental Biology 195:211-225

Minetti AE, Moia C, Roi GS, Susta D, Ferretti G. 2002. Energy cost of walking and running at extreme uphill and downhill slopes. Journal of Applied Physiology 93:1039-1046

Modica JR, Kram R. 2005. Metabolic energy and muscular activity required for leg swing in running. Journal of Applied Physiology 98:2126-2131

Moed B, Kram R. 2005. Metabolic costs of forward propulsion and leg swing at different running speeds. In: Proceedings from the XXth Congress of the International Society of Biomechanics. Cleveland State University, Cleveland. 
Nardello F, Ardigò LP, Minetti AE. 2011. Measured and predicted mechanical internal work in human locomotion. Human Movement Science 30:90-104

Pugh GC. 1971. The influence of wind resistance in running and walking and the mechanical efficiency of work against horizontal or vertical forces. Journal of Physiology 213:255-276 Robbins CT, Cohen Y, Davitt BB. 1979. Energy expenditure by elk calves. Journal of Wildlife Management 43:445-453

Roberts TJ, Kram R, Weyand PG, Taylor CR. 1998. Energetics of bipedal running. I. Metabolic cost of generating force. Journal of Experimental Biology 201:2745-2751

Roberts TJ, Marsh RL, Weyand PG, Taylor CR. 1997. Muscular force in running turkeys: the economy of minimizing work. Science 275:1113-1115

Rubenson J, Henry HT, Dimoulas PM, Marsh RL. 2006. The cost of running uphill: linking organismal and muscle energy use in guinea fowl (Numida meleagris). Journal of Experimental Biology 209:2395-2408

Smith NP, Barclay CJ, Loiselle DS. 2005. The efficiency of muscle contraction. Progress in Biophysics and Molecular Biology 88:1-58

Swanson SC, Caldwell GE. 2000. An integrated biomechanical analysis of high speed incline and level treadmill running. Medicine and Science in Sports and Exercise 32:1146-1155

Taylor CR, Caldwell SL, Rowntree VJ. 1972. Running up and down hills: some consequences of size. Science 178:1096-1097

Teunissen LPJ, Grabowski A, Kram R. 2007. Effects of independently altering body weight and body mass on the metabolic cost of running. Journal of Experimental Biology 210:4418-4427 Zani PA, Kram R. 2008. Low metabolic cost of locomotion in ornate box turtles, Terrapene ornata. Journal of Experimental Biology 211:3671-3676 


\section{Figure 1}

The total metabolic cost of running is comprised of several components

Parallel running refers to the task of running parallel to the surface whether that surface is level or inclined. The task of parallel running intrinsically requires bouncing perpendicular to the surface and that bouncing incurs a metabolic cost.

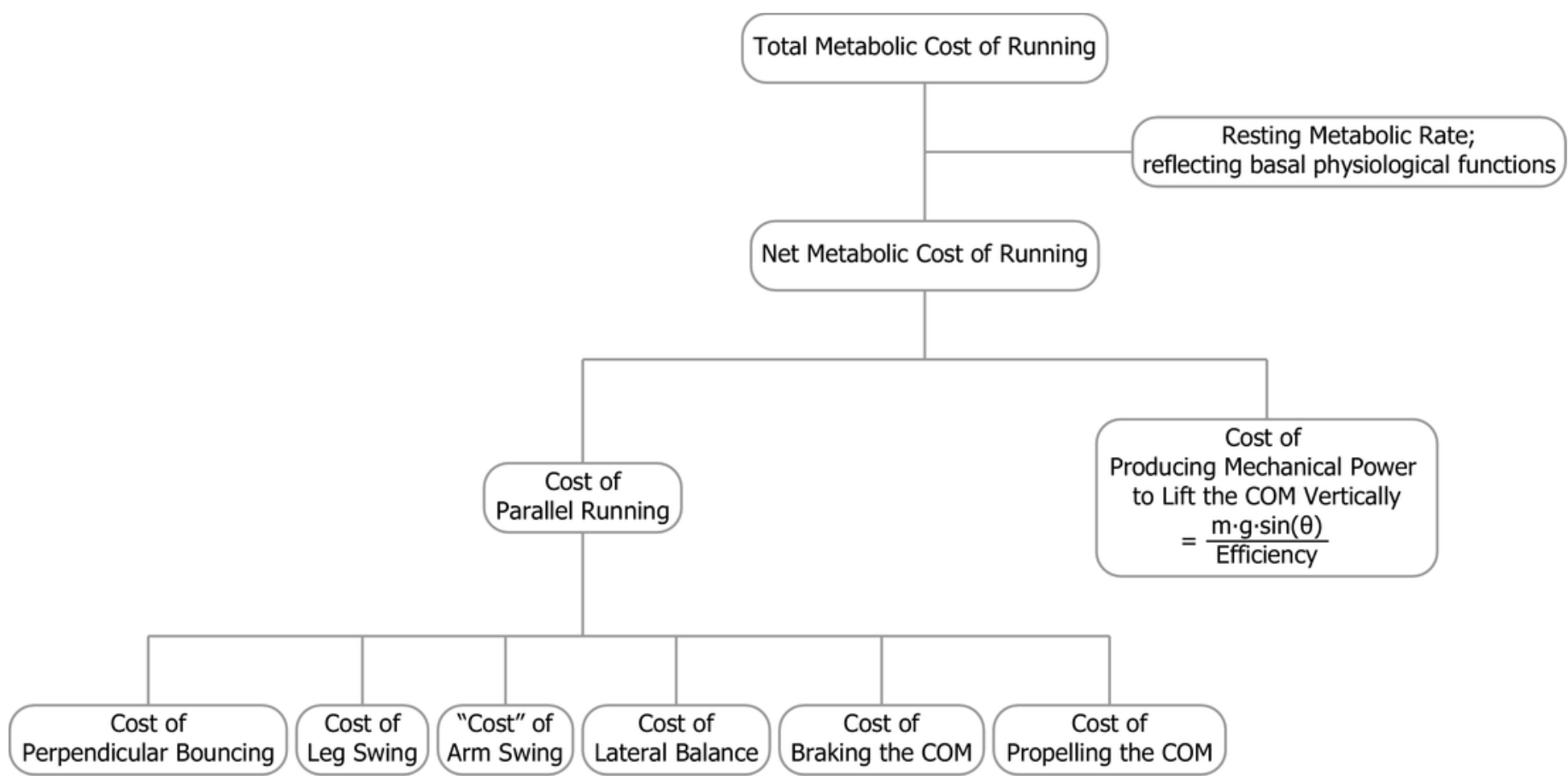




\section{Figure 2}

Ground reaction forces for different inclines

$A$ : Idealized parallel ground reaction force versus time traces for running at $3 \mathrm{~m} / \mathrm{s}$. $B$ : parallel component of gravitational impulse for a single step, and $C$ : schematic representation of the gravity force vector and its component parallel to the running surface. Forces are normalized to body weight.
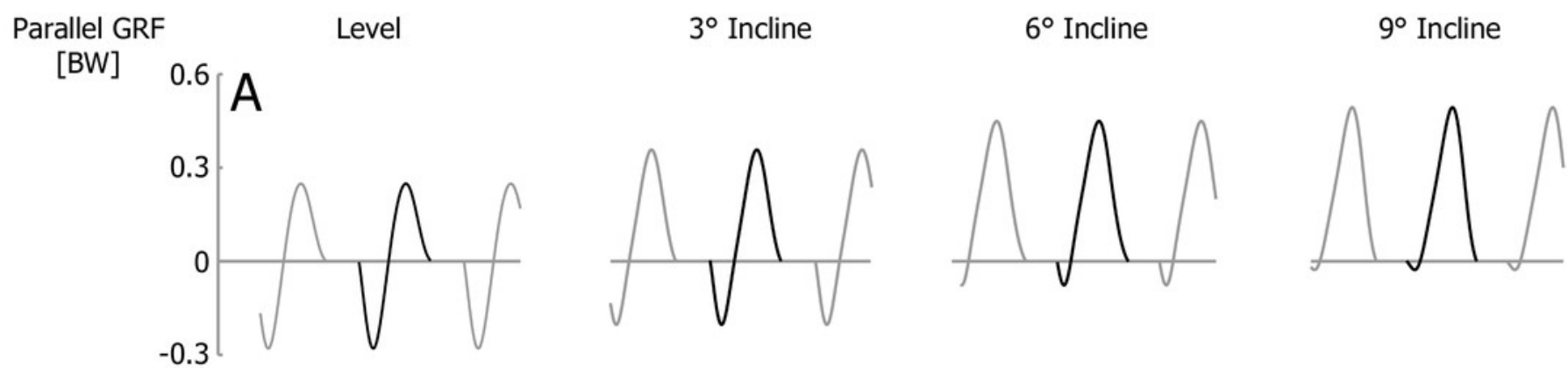

Parallel component gravitational force [BW]
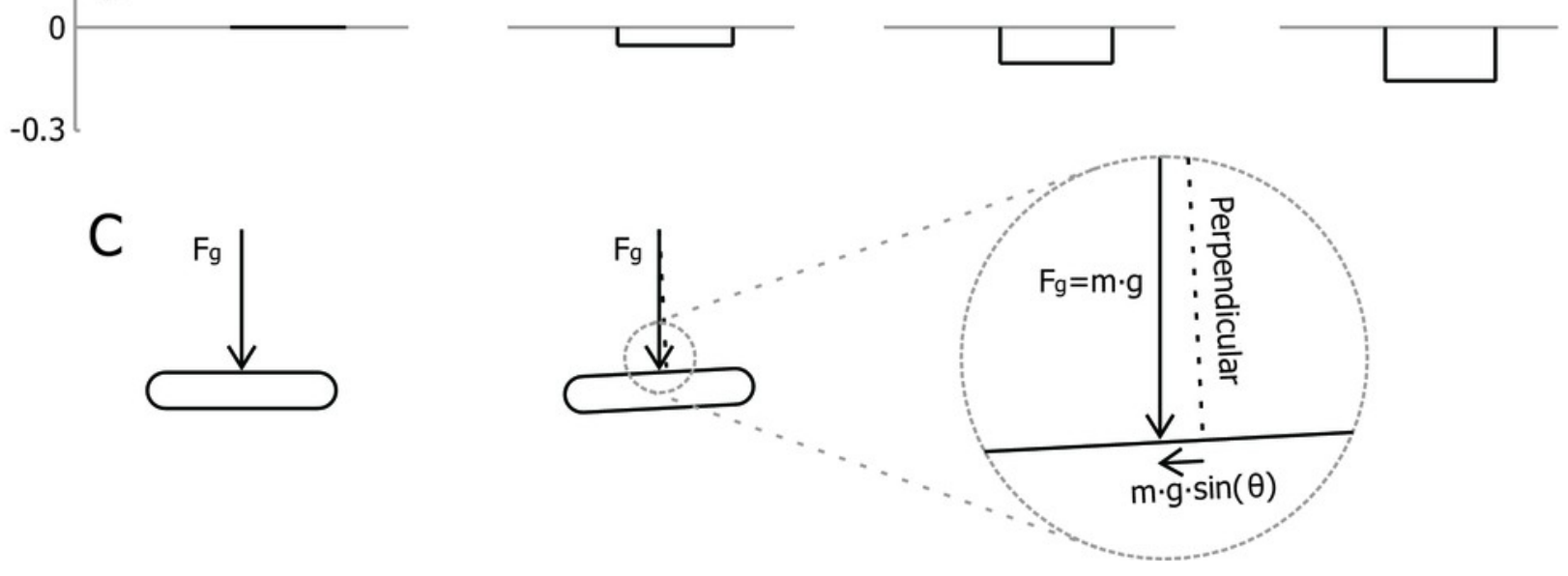


\section{Figure 3}

The wasted GRF impulse per step decreases for increasing inclines

Wasted GRF impulse for different inclines and velocities. Each small dot represents a single participant's wasted GRF impulse for a specific trial. The black line is the best-fit curve to the data according equation 6; the grey line is the best-fit linear equation. Note that the secondary horizontal axis at the top is the incline angle $\theta$, which is not linear, so the tick marks are not evenly spaced.
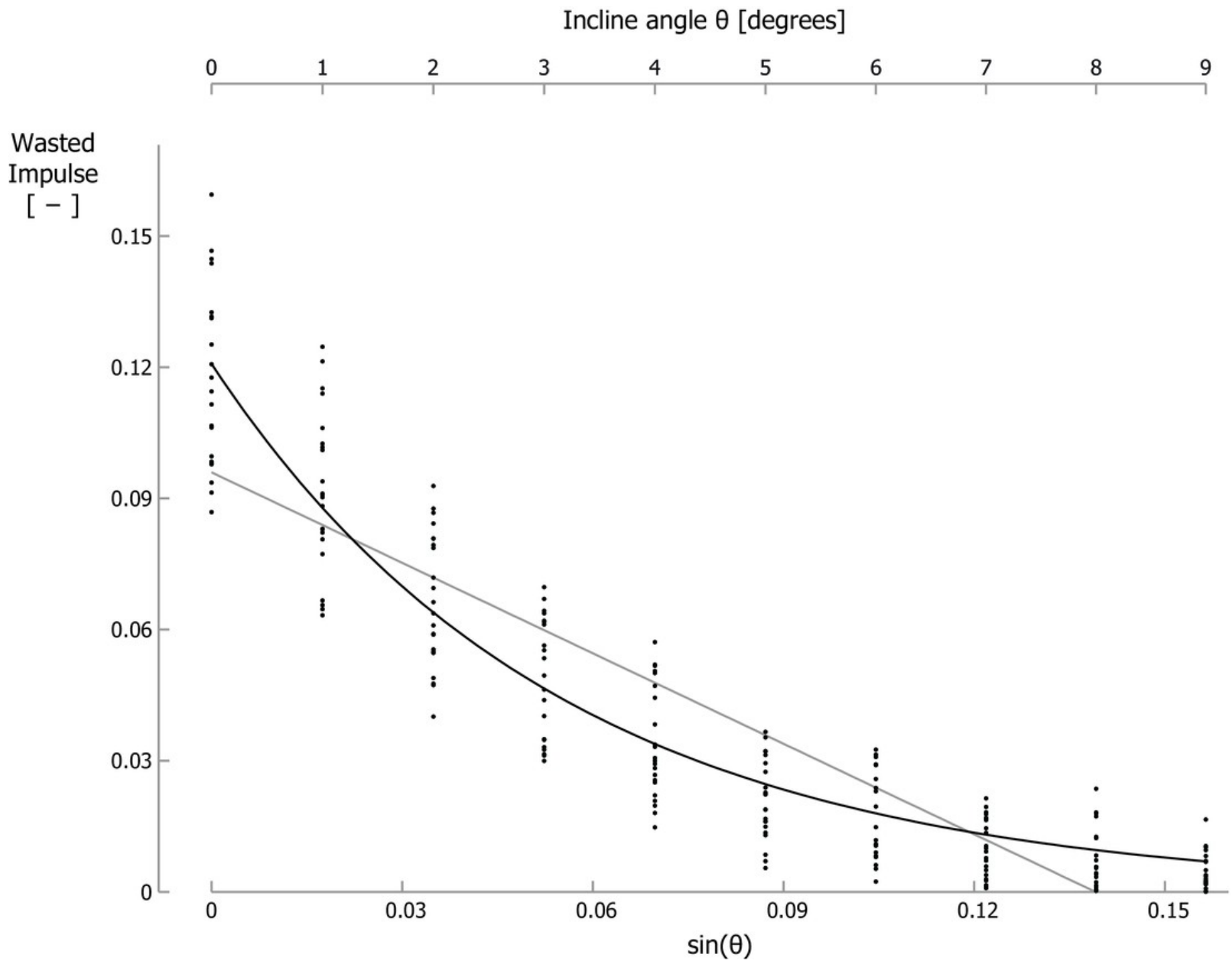


\section{Table 1 (on next page)}

Braking, total propelling and wasted impulses, step frequencies and contact times (mean \pm SD) for the different test conditions. 


\begin{tabular}{|c|c|c|c|}
\hline & $2.0 \mathrm{~m} / \mathrm{s}$ & $-63.9 \pm 11.9$ & $-1.7 \pm 1.7$ \\
\hline \multirow[t]{2}{*}{ Braking Impulse $\left(10^{-3}\right)$} & $2.5 \mathrm{~m} / \mathrm{s}$ & $-58.3 \pm 10.8$ & $-3.4 \pm 2.6$ \\
\hline & $3.0 \mathrm{~m} / \mathrm{s}$ & $-55.1 \pm 8.8$ & $-3.5 \pm 2.2$ \\
\hline \multirow{3}{*}{ Total Propelling Impulse $\left(10^{-3}\right)$} & $2.0 \mathrm{~m} / \mathrm{s}$ & $64.3 \pm 12.2$ & $0.9 \pm 1.3$ \\
\hline & $2.5 \mathrm{~m} / \mathrm{s}$ & $58.5 \pm 10.7$ & $2.0 \pm 2.5$ \\
\hline & $3.0 \mathrm{~m} / \mathrm{s}$ & $55.3 \pm 8.7$ & $2.3 \pm 2.1$ \\
\hline \multirow{3}{*}{$I \_G R F_{\text {wasted }}\left(10^{-3}\right)$} & $2.0 \mathrm{~m} / \mathrm{s}$ & $128.2 \pm 24.0$ & $2.6 \pm 2.9$ \\
\hline & $2.5 \mathrm{~m} / \mathrm{s}$ & $116.8 \pm 21.5$ & $5.4 \pm 5.0$ \\
\hline & $3.0 \mathrm{~m} / \mathrm{s}$ & $110.3 \pm 17.4$ & $5.7 \pm 4.2$ \\
\hline \multirow{3}{*}{ Step freqency (steps/minute) } & $2.0 \mathrm{~m} / \mathrm{s}$ & $2.68 \pm 0.15$ & $2.72 \pm 0.18$ \\
\hline & $2.5 \mathrm{~m} / \mathrm{s}$ & $2.78 \pm 0.20$ & $2.84 \pm 0.16$ \\
\hline & $3.0 \mathrm{~m} / \mathrm{s}$ & $2.84 \pm 0.19$ & $2.94 \pm 0.16$ \\
\hline \multirow{3}{*}{ Contact times (s) } & $2.0 \mathrm{~m} / \mathrm{s}$ & $0.31 \pm 0.03$ & $0.32 \pm 0.03$ \\
\hline & $2.5 \mathrm{~m} / \mathrm{s}$ & $0.28 \pm 0.03$ & $0.28 \pm 0.03$ \\
\hline & $3.0 \mathrm{~m} / \mathrm{s}$ & $0.25 \pm 0.02$ & $0.25 \pm 0.02$ \\
\hline
\end{tabular}




\section{Table 2 (on next page)}

Measured rates of oxygen consumption (VO2) and metabolic rates (mean \pm SD) for the different test conditions. 


\begin{tabular}{|c|c|c|c|c|c|}
\hline Day & $\begin{array}{l}\text { Velocity } \\
(\mathrm{m} / \mathrm{s})\end{array}$ & $\begin{array}{c}\text { Angle } \\
\text { (degrees) }\end{array}$ & $\begin{array}{c}\text { Grade } \\
(\%)\end{array}$ & $\begin{array}{c}\mathrm{VO}_{2} \\
(\mathrm{ml} /(\mathrm{kg} \cdot \min ))\end{array}$ & $\begin{array}{c}\text { Metabolic rate } \\
(\mathrm{W} / \mathrm{kg})\end{array}$ \\
\hline \multirow{8}{*}{1} & Standing & - & - & $4.3 \pm 0.5$ & $1.5 \pm 0.1$ \\
\hline & & 0 & 0 & $24.5 \pm 1.5$ & $8.3 \pm 0.4$ \\
\hline & & 1 & 1.7 & $26.1 \pm 1.2$ & $8.9 \pm 0.4$ \\
\hline & & 2 & 3.5 & $29.1 \pm 1.3$ & $9.9 \pm 0.4$ \\
\hline & 2.0 & 3 & 5.2 & $31.6 \pm 1.8$ & $10.8 \pm 0.5$ \\
\hline & & 4 & 7.0 & $34.3 \pm 1.1$ & $11.7 \pm 0.4$ \\
\hline & & 6 & 10.5 & $40.5 \pm 1.8$ & $13.9 \pm 0.6$ \\
\hline & & 8 & 14.1 & $47.1 \pm 2.1$ & $16.3 \pm 0.7$ \\
\hline \multirow{11}{*}{2} & Standing & - & - & $4.7 \pm 0.4$ & $1.6 \pm 0.1$ \\
\hline & & 0 & 0 & $29.0 \pm 1.3$ & $9.8 \pm 0.4$ \\
\hline & & 1 & 1.7 & $31.6 \pm 1.4$ & $10.7 \pm 0.4$ \\
\hline & 2.5 & 2 & 3.5 & $35.4 \pm 1.4$ & $12.0 \pm 0.4$ \\
\hline & & 3 & 5.2 & $38.3 \pm 1.2$ & $13.1 \pm 0.4$ \\
\hline & & 4 & 7.0 & $42.2 \pm 1.0$ & $14.4 \pm 0.3$ \\
\hline & & 0 & 0 & $35.3 \pm 1.6$ & $11.9 \pm 0.5$ \\
\hline & & 1 & 1.7 & $38.9 \pm 2.0$ & $13.2 \pm 0.6$ \\
\hline & 3.0 & 2 & 3.5 & $43.1 \pm 1.8$ & $14.7 \pm 0.6$ \\
\hline & & 3 & 5.2 & $47.1 \pm 1.7$ & $16.1 \pm 0.5$ \\
\hline & & 4 & 7.0 & $51.6 \pm 2.2$ & $17.8 \pm 0.7$ \\
\hline
\end{tabular}




\section{Figure 4}

Net metabolic Cost of Transport

A: Net metabolic Cost of Transport (CoT) for different inclines and velocities. CoT is the net metabolic energy consumed per meter traveled parallel to the running surface. Each small dot represents a single participant's CoT for a specific trial. The black line is the best-fit curve of the net CoT according equation 8. Note that the secondary horizontal axis at the top is the incline angle $\theta$, which is not linear, so the tick marks are not evenly spaced. $B$ : Net metabolic $\mathrm{CoT}_{\text {total }}$ and metabolic CoT $\mathrm{T}_{\text {vertical. }}$ : : Metabolic CoT of parallel running. The grey line represents the constant CoT components of parallel running (perpendicular bouncing, leg and arm swing and lateral balance); the remainder, the CoT of braking and propelling, approaches zero at steeper inclines. Symbols represent mean values: $\bigcirc=2.0 \mathrm{~m} / \mathrm{s}, \Delta=2.5$ $\mathrm{m} / \mathrm{s}, \square=3.0 \mathrm{~m} / \mathrm{s}$.

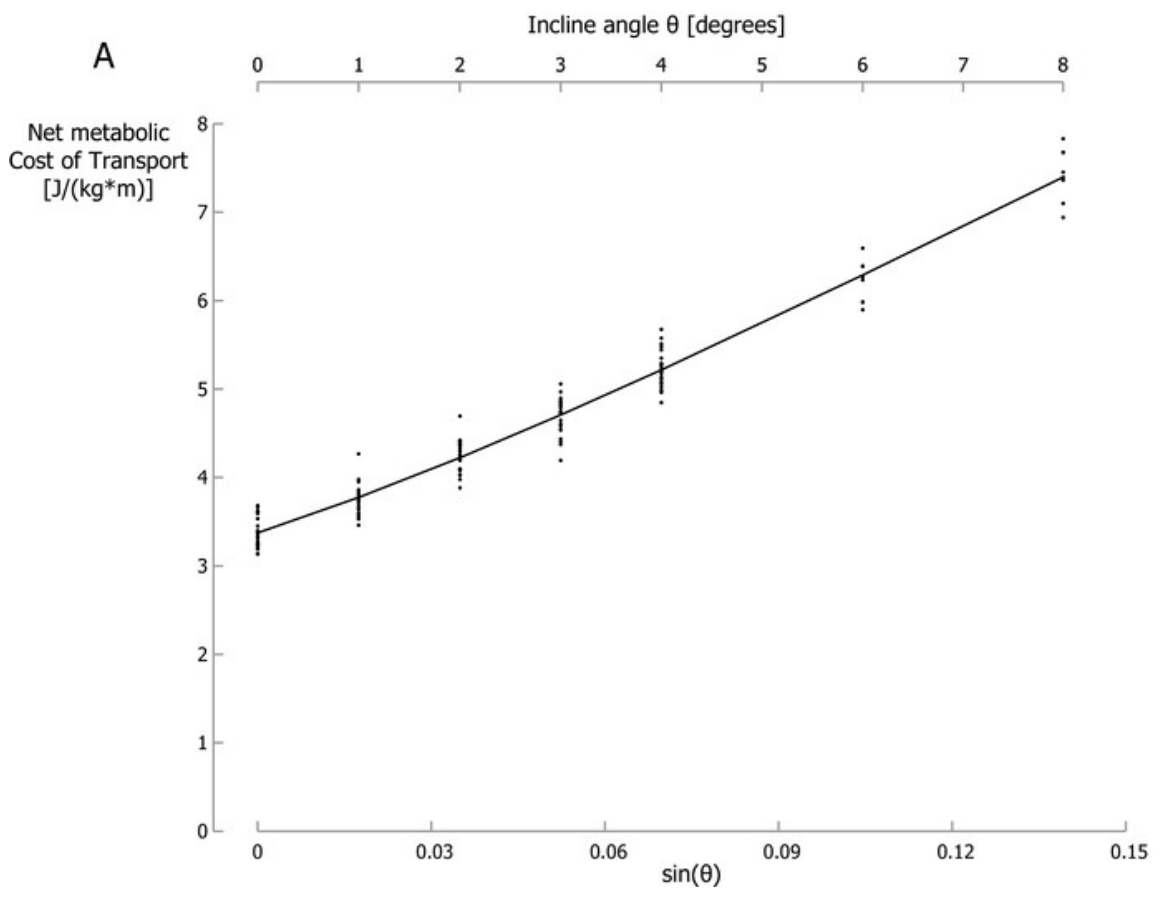

B

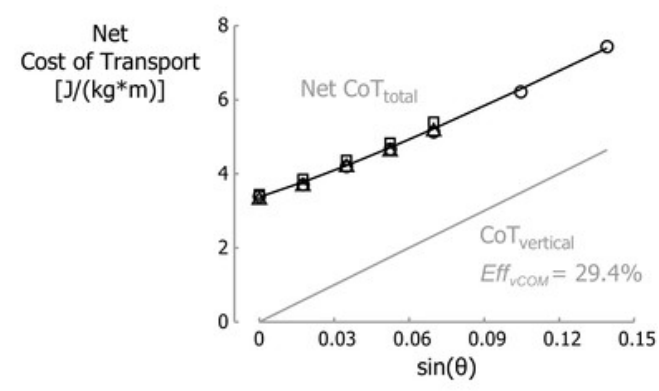

C

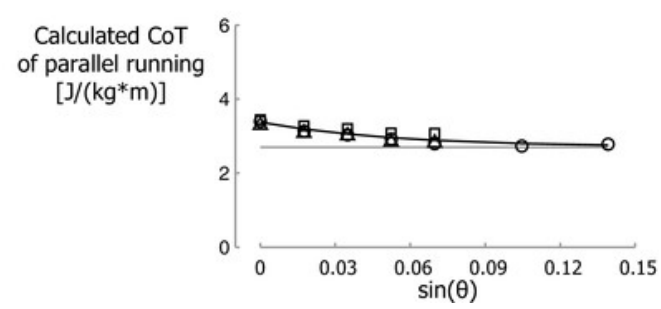

